\title{
Exploring HTTP Header Manipulation In-The-Wild
}

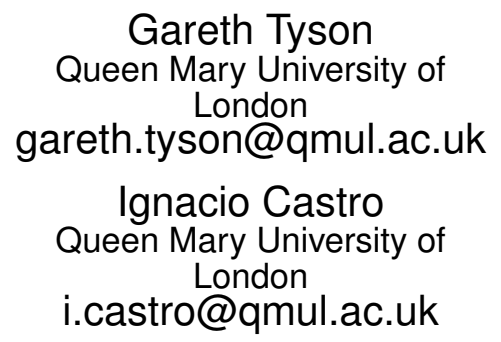

Gareth Tyson

London

gareth.tyson@qmul.ac.uk

Ignacio Castro

i.castro@qmul.ac.uk

\author{
Shan Huang \\ Queen Mary University of \\ London \\ shan.huang@qmul.ac.uk \\ Vasile C. Perta
Sapienza University of Rome
perta@di.uniroma1.it
}

\author{
Steve Uhlig \\ Queen Mary University of \\ London \\ steve.uhlig@qmul.ac.uk
}

\author{
Felix Cuadrado \\ Queen Mary University of \\ London \\ felix.cuadrado@qmul.ac.uk
}

Arjuna Sathiaseelan

University of Cambridge

arjuna@cl.cam.ac.uk

\begin{abstract}
Headers are a critical part of HTTP, and it has been shown that they are increasingly subject to middlebox manipulation. Although this is well known, little is understood about the general regional and network trends that underpin these manipulations. In this paper, we collect data on thousands of networks to understand how they intercept HTTP headers in-the-wild. Our analysis reveals that 25\% of measured ASes modify HTTP headers. Beyond this, we witness distinct trends among different regions and AS types; e.g., we observe high numbers of cache headers in poorly connected regions. Finally, we perform an in-depth analysis of the types of manipulations and how they differ across regions.
\end{abstract}

\section{INTRODUCTION}

HTTP underpins one of the most successful inventions in recent history: the World Wide Web. Although HTTP has received much attention, an aspect that remains understudied is that of headers. These are attribute-value pairs that are embedded within all HTTP messages. While they are well documented within standards, little is known of their practical usage at scale. This is exacerbated by the increasing propensity for middleboxes to manipulate headers (e.g., for caching, monitoring, censorship). While it is known that users' private information can be exposed and tracked through middleboxes [5], we posit that such middlebox injections may also reveal a wealth of information about the networks engaged in the header manipulation. We therefore ask which insights can be extracted from header manipulations performed across regions and networks?

To gain an understanding of the use of HTTP headers, we begin by collecting data on almost 1 million websites (\$2). We then present a novel methodology to collect large-scale data on global HTTP middlebox header manipulation. This involves creating a

(C) 2017 International World Wide Web Conference Committee (IW3C2), published under Creative Commons CC BY 4.0 License. WWW 2017, April 3-7, 2017, Perth, Australia. ACM 978-1-4503-4913-0/17/04. http://dx.doi.org/10.1145/3038912.3052571

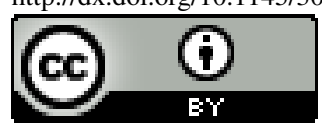

measurement platform using the Hola peer-to-peer proxy network [2] (\$3). Using this platform, we craft and forward HTTP requests via third party networks to a web server we control. By monitoring both the request and response endpoints, we can discover manipulations performed by these networks. Exploiting Hola, we launch over 400k HTTP queries from 143k vantage points in $3818 \mathrm{Au}-$ tonomous Systems (ASes) - one of the largest studies of its kind. Unlike techniques using controlled infrastructures (e.g., Planetlab), this provides unique visibility on a range of network types in countries rarely studied, e.g., over 400 ASes in Africa $(\S 4)$.

In this paper we explore the propensity of different network types and regions to manipulate HTTP headers, in terms of both frequency $(\S 5)$, and content $(\S 6)$. We find that header manipulation is remarkably widespread: hosts in $25 \%$ of measured ASes witness header modifications at least once. Despite this headline figure, our data shows that the density of middlebox injections varies dramatically across regions, with networks in technologically advanced countries abandoning the use of caches.

A common theme in our findings is the lack of standards adherence; we find thousands of new non-standard headers returned by web servers $(\$ 2)$, alongside networks injecting over 40 nonstandard headers $(\$ 6)$. Although this form of extensibility could be considered desirable, our findings suggest that it runs the risk of bloating the protocol in a way that breaks down common understanding. For example, amongst other things, we observe middleboxes injecting cookies, disabling performance-enhancing features, caching against our dictates and adding private information into requests.

Opinion on whether or not this is damaging is divided, however, we believe that the Web community is at a juncture at which they should decide if they wish to support (e.g., via mcTLS [20]) or undermine this behaviour. This is particularly important considering recent efforts towards new web protocol standards $[8,13]$. As such, this paper provides a unique insight into in-the-wild practices, and how future protocol decisions on header manipulation may affect operators in different regions.

\section{A PRIMER ON HTTP HEADERS}

Before studying middlebox header interference, it is important to understand ( $i$ ) what HTTP headers are, and (ii) how they are currently used by web servers. HTTP was developed in 1991 with the advent of HTTP 0.9. Unlike subsequent versions, HTTP 0.9 was 


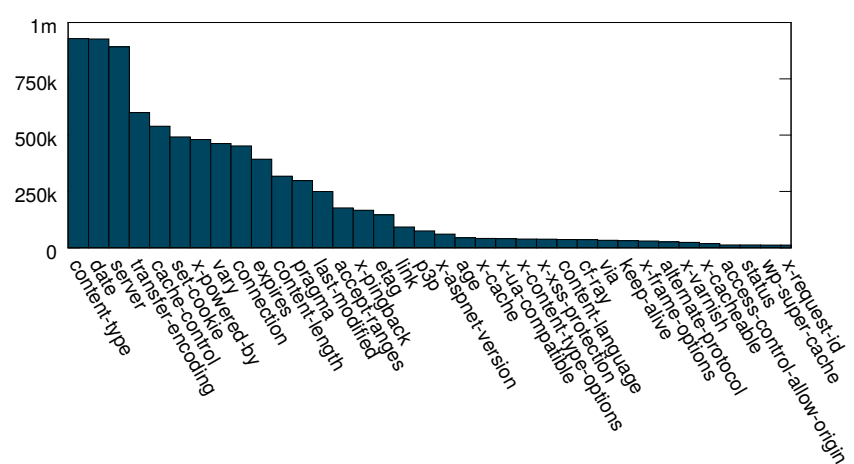

Figure 1: Most popular response headers returned from Alexa 1 Million websites. Y axis is number of websites.

an extremely simple protocol consisting of just single line requests. As webpages increased in sophistication, it became desirable for further information to be exchanged between clients and servers. To address this need, HTTP 1.0 introduced headers. HTTP headers are text-based attribute-value pairs set by clients and servers. These allow the two end points to exchange metadata regarding requests and responses. For instance, standard headers in HTTP 1.1 allow servers to inform clients about content encoding, caching times-tolive, as well as websites that clients should be redirected to. Once headers are transmitted across a network, though, they become vulnerable to manipulation by intermediate parties. This is enabled by the remaining bulk of non-encrypted HTTP traffic [19].

To briefly get an idea of the headers used by servers and how they might differ from those injected by middleboxes, we have scraped the Alexa Top 1 Million websites to collect their response headers. We filtered any unsuccessful fetches after two separate attempts, leaving 928,724 websites. The majority run HTTP 1.1 , however, almost 10k websites are still using HTTP 1.0 (despite being deprecated over 15 years ago). Websites, on average, have 9.88 headers, with a propensity for higher ranked websites to increase this (e.g., 14 for Google, 19 for Facebook, 22 for Twitter). Notable outliers exist too; for instance, pokoopka.com has 230 (repeated) Set-Cookie headers, while the website with the largest number of unique headers is tiempy. com (with 45). This website has apparently nonsensical headers (it spells out words across multiple headers). There are many examples of these unusual configurations. In total, there are 97 websites that have in excess of 50 (non-unique) headers, and 2252 with more than 25. Standard head$\mathrm{ers}^{1}$ make up the bulk (84.6\%), although there are far more unique non-standard headers than known headers. Half of the 9899 unique header attributes seen in HTTP 200 OK responses occur only once in the dataset, indicating that they are specific to individual website deployments. Figure 1 presents a histogram of the most frequently seen headers. It is beyond the scope of this paper to delineate them, but it can be seen that certain blocks of standard headers are frequently seen; e.g., Content-Type, Server and Date are returned by over $95 \%$ of websites. A long-tail then emerges with non-standard and vendor-specific headers being used by smaller numbers of sites, e.g., CF-RAY (used by CloudFlare) and WPSuper-Cache (used by WordPress). We even witness job adverts and jokes contained within headers (e.g., X-Hacker). Adherence to standards is clearly not considered critical. We take this as strong motivation to look at the other ways in which these standards may be undermined.

\footnotetext{
${ }^{1}$ As dictated by IANA [17].
}

\section{METHODOLOGY AND DATASET}

\subsection{Overview of Hola}

We begin by briefly describing Hola, which we use to launch our measurements [3]. Hola is a peer-to-peer proxy network that allows clients to forward their requests through other peers running the Hola-browser plugin. This allows clients to appear as if their requests are emanating from different networks (often to avoid geofirewalls). Hola consists of a mix of dedicated servers and peers running a local browser plugin. When a client wishes to proxy a web fetch through another country, it sends its request via a Hola server called a zagent. Each zagent runs multiple proxy processes on different ports, with each port dedicated to forwarding requests via a given country. Upon receiving a web request, these zagents forward the request via a peer in the appropriate country.

\subsection{Methodology}

\subsubsection{Data Collection}

We use Hola to trigger HTTP requests from third party networks around the globe towards a web server we control. Hola simply forwards the requests we send it, allowing us to craft the exact HTTP request headers we desire. By crafting both the request and response, we can detect any header modifications taking place in the networks through which Hola forwards the traffic. We selected Hola as we wish to gain a wider vantage than that provided by academic networks (e.g., Planetlab) or data centre networks (e.g., open proxies [23]). This diversity is later confirmed ( $c f$. §4).

We use a default Apache web server build (7 response headers) with the exception of disabling all caching (using the CacheControl header). This configuration reflects well the typical setups we observe in our Alexa scrapes $(\$ 2)$. We then iterate through all country codes, requesting that Hola forwards our request through each country one-by-one. In this round-robin fashion, we launched 405k HTTP GET requests to our server via Hola (using their API). Our requests contained 6 default headers: Host, User-Agent, Accept, Keep-Alive, Accept-Encoding and Connection. We record all messages received at our client and server side, after having been proxied through Hola.

\subsubsection{Data Processing \& Cleaning}

We next subset our data to leave only successful fetches. We then separate our dataset into requests (received by our server) and responses (received by our client). Both have been routed through a third party network using Hola. We consider a request/response as being modified if any of the following cases apply: $(i)$ the value of a header has had one or more character modifications; (ii) a header attribute has been added; or (iii) removed.

This allows us to see if a request or response is modified. A key issue, however, is minimising the possibility of Hola peers locally manipulating our headers (e.g., by anti-virus software). To mitigate this, we manually identify headers that are known to be injected by software running on end hosts. This was done using a range of online resources, e.g., IANA, RFCs, web security services. This revealed 5 locally injected headers in our data. All such headers were filtered from the data. This cleaning removed 368 instances of local response header injection, and 3 instances of request header injection. The overwhelming majority of these instances (98\%) were generated by malware and adware running on the Hola peer: XOSSProxy, Gyoarazujo and X-Vitruvian. These operate as local proxies that intercept and manipulate requests (e.g., to inject adverts). The remaining $2 \%$ were less nefarious, e.g., ad blockers and Do Not Track. 
This still leaves the small possibility that some end hosts were injecting headers traditionally injected by in-network middleboxes (e.g., by installing Squid locally). To identify this, we separated all samples into their origin ASes and extracted ASes for which we have over 5 samples. We then searched for peers that were the only nodes in the AS to make a given change. The rationale was that such peers may be injecting headers locally, thereby differentiating themselves from the remaining samples in the AS. We then manually inspected these manipulations, discovering the presence of various headers containing things like localhost or 127.0.0.1. Hence, we compiled a simple set of rules to filter such fetches from the dataset (removing 1016 samples). Following this step, out of $405 \mathrm{k}$ fetches, only 88 requests and 92 responses remained that were exclusively manipulated by a single node in an AS. These showed no explicit indications of local manipulation. We are therefore confident that any remaining modifications were introduced somewhere along the intermediate peer $\rightarrow$ server path and not by the peer itself. Our data is available at [26].

\subsubsection{Peer Metadata}

To get an idea of the networks we have sampled, we augment each Hola peer with metadata. First, we geolocate every peer IP address. It is well known that individual geolocation databases contain errors [21]. Hence, we use majority voting from 10 separate databases. ${ }^{2}$ These IPs correspond to either the public address of the peer or, alternatively, an intermediate TCP-terminating middlebox (generally these are in the same network, but not always). Further, we limit our granularity to country-level geolocation, which reports high accuracy [24]. We then map each peer to their respective AS using the same databases. Finally, we tag each peer with its AS type. We experimented with three off-the-shelf AS classifiers: (i) The CAIDA AS Rankings [1]; (ii) A classifier provided by Dhamdhere et al. [11]; and (iii) A classifier provided by Dimitropoulos et al. [12]. The first provided high recall, but only a coarse (3 category) classification, whilst the other two provided much lower recall but a slightly finer-grained classification. In this paper we utilise the third classifier, as this offered similar recall to (ii) but with a finer grained classification.

\subsection{Data Limitations and Ethics}

It is important to outline key limitations in the dataset. Most obvious is the use of a third party system (Hola), which could introduce unexpected behaviour. To mitigate this risk, we have extensively tested Hola to identify unusual behaviour. Fortunately, the only anomalies seen (e.g., injecting X-Hola-Error headers) can be cleaned from the data. Another consideration is that our measurements are not an unbiased sample of networks around the globe. As a general-purpose tool, the bias induced by more Western "techsavvy" crowd sourced measurement platforms may be reduced though. We also acknowledge that Hola cannot tell us where the manipulations happen. That said, by filtering locally injected headers, we can be confident that any modifications were performed on the peer $\rightarrow$ server path. Of course, this leaves the possibility that the middlebox exists in a different network to the client, however, this still reveals a network that is subjected to manipulations. Hence, we temper our analysis with this consideration. Finally, it is worth noting that the use of Hola may raise ethical questions, as we are forwarding requests through users' machines. To mitigate these, we only forward requests to our own web server (containing a "Hello

\footnotetext{
${ }^{2}$ OpenIPMap; MaxMind; Whois; RIRs' allocation files for RIPE, APNIC, ARIN, AFRINIC and LACNIC; Team Cymru; and Reverse DNS lookups, which were used to infer the location based on city, country codes $(\mathrm{CCs})$ or airports in the reverse names.
}

World" page). As such, there is no risk of triggering censorship. Further, Hola informs its users about how it operates. Hence, users are already aware that they operate as proxy points. We have obtained IRB approval.

\section{CHARACTERISING HOLA}

We begin by briefly describing Hola's scale and distribution, as captured by our measurements. In total, we have collected data covering 143,288 IP addresses in 3818 ASes. To add context, this can be compared against other state-of-the-art measurement platforms, e.g., Dasu (2431 ASes [22]), although it should be noted that each platform supports very different features. Figure 2 presents the density of (i) Hola users, as measured by IP addresses; and (ii) unique ASes hosting Hola nodes. It can be seen that Hola possesses vantage on every region in the world: 216 countries and territories. Consequently, the beauty of Hola, compared to other platforms, is that is can provide very wide vantage on these global users. This is an attractive property, as we see data regarding many areas that often are excluded from such studies (e.g., Africa). In terms of IP addresses, the best represented country is the US, whereas, in terms of ASes, Ukraine is best represented. At first, we thought this may be a geolocation error, however, we manually confirmed the veracity. This is perhaps a product of the differing ISP market structures seen in these countries. But, to address this imbalance, we primarily characterise manipulations on a per-AS basis (rather than per-IP), to reduce the bias introduced by highly populous ASes.

To better understand the nature of Hola's network sampling, Table 1 provides a breakdown of the types of networks seen (based on the classifier in \$3.2.3). It only lists statistics on the ASes that were successfully classified. Customer indicates a variety of commercial networks that do not offer residential Internet access (e.g., web hosts), whilst Network Information Centres (NICs) are ASes that host important infrastructure (e.g., root domain name servers). Full details of the AS meanings and methodology can be found in [12]. The analysis confirms a wide sample of networks types. We argue this diversity is highly attractive for studying header manipulations, as it gives a wide vantage on global behaviour. More generally, this also confirms Hola's efficacy as a powerful platform for web data collection.

\section{WHO MANIPULATES HEADERS?}

\subsection{Measuring Across Networks}

First, we inspect how header manipulations occur across the sampled ASes. $21 \%$ of the ASes have requests manipulated, compared to $19 \%$ for responses. Overall, $25 \%$ contain sessions that manipulate headers at least once. We discover that classifying ASes in this manner, however, is not straightforward. To highlight this, Figure 3 presents the top 100 most sampled ASes that contain modifications to headers; each AS is separated into requests that were manipulated and requests that were not. Curiously, it can be seen that many ASes contain both modified and non-modified requests. Evidently, this is problematic when classifying an entire AS. Inspection suggests that this occurs for a variety of reasons, most likely due to a diversity of paths and middlebox deployments within a large AS and its interconnected networks (as well as misconfiguration [25]).

This also raises the question of what types of ASes witness these changes. To answer this, we separate all ASes into the classifications presented in \$3.2.3. Figure 4 breaks down ASes into their types, and then presents CDFs of the percentage of samples in each AS that modify headers. Table 1 further provides statistics on the 


\begin{tabular}{|c|c|c|c|c|c|c|c|c|c|c|c|}
\hline \multirow[t]{2}{*}{ AS Classification } & \multirow[t]{2}{*}{ \#ASes } & \multicolumn{2}{|c|}{$\begin{array}{l}\text { \#Samples } \\
\text { Modified }\end{array}$} & \multicolumn{2}{|c|}{$\begin{array}{l}\text { \#Samples } \\
\text { Not Modi- } \\
\text { fied }\end{array}$} & \multicolumn{2}{|c|}{$\begin{array}{l}\text { \%Samples } \\
\text { modified }\end{array}$} & \multicolumn{2}{|c|}{$\begin{array}{l}\% \text { ASes that } \\
\text { modify at least } \\
\text { once }\end{array}$} & \multicolumn{2}{|c|}{$\begin{array}{l}\text { Mean \% Sam- } \\
\text { ples Modified } \\
\text { Per AS }\end{array}$} \\
\hline & & Req & Res & Req & Res & Req & Res & Req & Res & Req & Res \\
\hline Net Info Centre & 41 & 992 & 585 & 8059 & 8466 & 10.96 & 6.46 & 34.15 & 39.02 & 7.39 & 7.50 \\
\hline Customer & 348 & 2432 & 978 & 29086 & 30536 & 7.72 & 3.10 & 15.52 & 15.80 & 5.58 & 4.72 \\
\hline Regional ISP & 798 & 9395 & 4184 & 159589 & 164798 & 5.56 & 2.48 & 32.08 & 29.82 & 7.06 & 4.40 \\
\hline Tier-1 & 15 & 47 & 21 & 2998 & 3024 & 1.54 & 0.69 & 26.67 & 33.33 & 10.33 & 7.38 \\
\hline University & 62 & 9 & 7 & 1009 & 1011 & 0.88 & 0.69 & 4.84 & 3.23 & 1.97 & 1.94 \\
\hline
\end{tabular}

Table 1: Statistics per AS types (excluding samples that could not be classified into AS type).

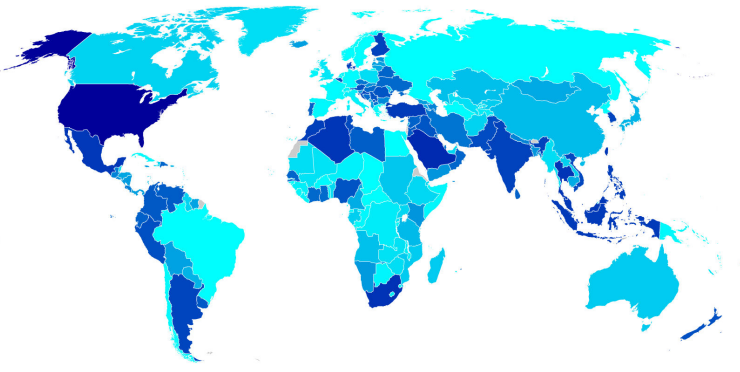

(a) Number of IP addresses per country ( $\max 2803)$

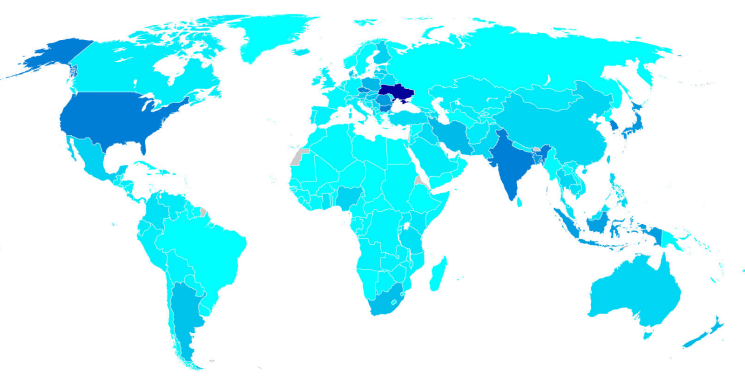

(b) Number of ASes per country (max 325)

Figure 2: Map of IP and AS samples from Hola; dark blue represents highest density.

behaviour of ASes within each group. It can be seen that the different AS types exhibit a range of trends. University ASes stand out as having very few modifications (3\% of ASes for responses, $5 \%$ for requests). Overall, networks classified as NICs are the most likely to manipulate headers (39\% of ASes for responses, $34 \%$ requests). While it is hard to definitively state the reasons, it is well known that university networks tend not to transparently intercept traffic [10]. NICs and hosting centres, on the other hand, may deploy dedicated infrastructure to optimise their activities. It can similarly be seen that ISPs tend to have high proportions of manipulations. $32 \%$ of regional ISPs inject headers into requests at least once. Again, this reflects commonly understood business models, in which these edge networks may wish to reduce their egress traffic by utilising cache middleboxes. Studying middleboxes without an appreciation of the types of networks sampled therefore could be quite misleading.

The above indicates that networks might deploy header-manipulating middleboxes in only a subset of locations. To expand on this, we can also check if the manipulated samples seen within an AS always make the same modifications. To explore this, we subset samples to leave only those in which we detect manipulation. We

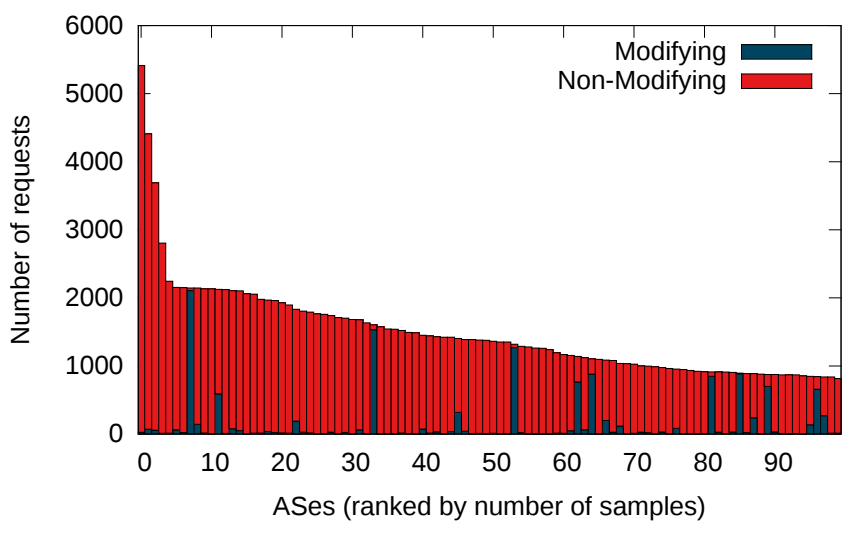

Figure 3: Number of requests for the top 100 ASes sampled that modify headers at least once (manipulated vs. not manipulated samples per AS) .

then map them into their respective ASes, and filter out any ASes in which we only have a single IP address. For each AS, $i$, we extract the full set of headers that are manipulated, $H^{i}$. For each header, $h \in H^{i}$, we calculate the percentage of samples in the AS where $h$ is modified. For example, if all samples in an AS modify the Server header, we assign that AS a Server value of 100\%. By averaging the percentages within an AS for all manipulated headers, $\bar{H}^{i}$, a single value can be obtained per-AS. Figure 5 presents a CDF of the per-AS averages. It can be seen that only $44 \%$ of ASes exclusively record the same modifications for all requests, whilst this is $32 \%$ for responses. This leaves a significant fraction of samples from an AS that vary the headers that are manipulated. This confirms that users in individual ASes are subject to a mix of manipulations, and the heteogeneity of these changes is high.

\subsection{Measuring Across Regions}

Anecdotally, it is known that different regions have different styles of network deployment. With our data it is possible to evaluate the prevalence of header-manipulating middleboxes across different regions. We sample all continents: Africa (442 ASes), Asia (1114), Europe (1581), Middle East (209), North America (394), Oceania (154) and South/Central America (182). ${ }^{3}$ Figure 6 presents the fraction and number of ASes per country (top 30 countries) that record manipulated headers. Absolute numbers are shown with the bars (right axis), whilst the fraction of modifying ASes is shown by the line (left axis). We only include countries where we have sufficient sampling, of at least 10 ASes. Broadly speaking, we see sim-

\footnotetext{
${ }^{3}$ Note that some ASes have a presence in multiple continents, and therefore these samples are spit between regions.
} 


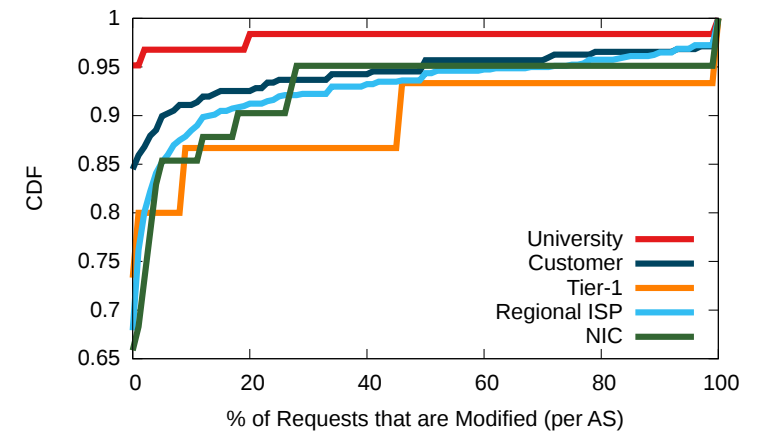

Figure 4: CDF of the percentage of requests that are modified on a per AS basis. Data is separated into network types.

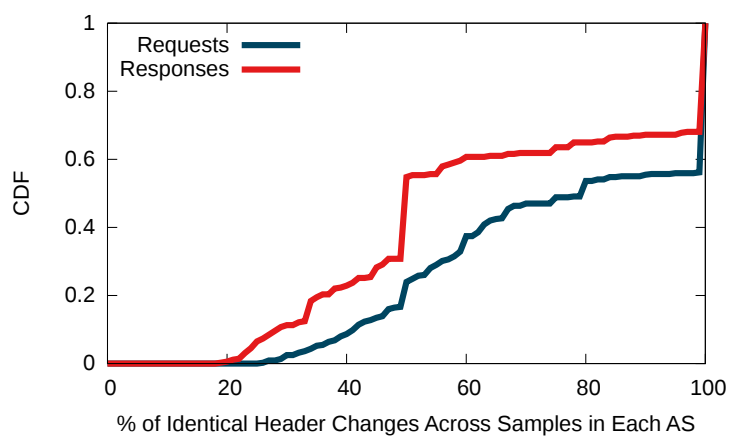

Figure 5: CDF of percentage of Hola peers in an AS that see the same headers changed. Each AS is represented by the average taken across all headers manipulated.

ilar fractions for both request and response manipuation per country (correlation 0.73 ), however, there are noteworthy discrepancies, e.g., in Jordan, only $21 \%$ of ASes manipulate responses, compared to $50 \%$ that manipulate the requests. This confirms the need to use both endpoints to detect interference.

The reasons for these countries exhibiting such trends are likely diverse. To get an idea of these, we compare them against well known technological, economic and societal measures. To this end, we collect metrics from $(i)$ The Web Index: a composite ranking that measures a country's online capabilities; and (ii) The World Bank: an organisation that compiles economic data. Figure 7 presents the Spearman correlation coefficient for the fraction of ASes that manipulate headers in a given country vs. the metrics taken from the Web Index and the World Bank. Due to space constraints, we do not delineate the nature and methodology behind each metric; instead, full details can be found at [31, 32]. However, it can be seen that there is a strong negative correlation between a country's header-manipulating middlebox deployment and its positions in these metric rankings. High middlebox deployments are correlated with less developed countries (as measured via the metrics presented). This can generally be observed too, e.g., $36 \%$ of African ASes manipulate requests compared to just 8\% in Europe. This is somewhat counterintuitive as one might expect more developed countries to have larger infrastructure deployments. The most correlated factors in Figure 7 pertain to how widely available services are to individuals in a country. It appears that nations with less developed infrastructures rely more heavily on the use of middleboxes (most prominently caches). To explain this, we contacted several operators in both Europe and Africa, who confirmed

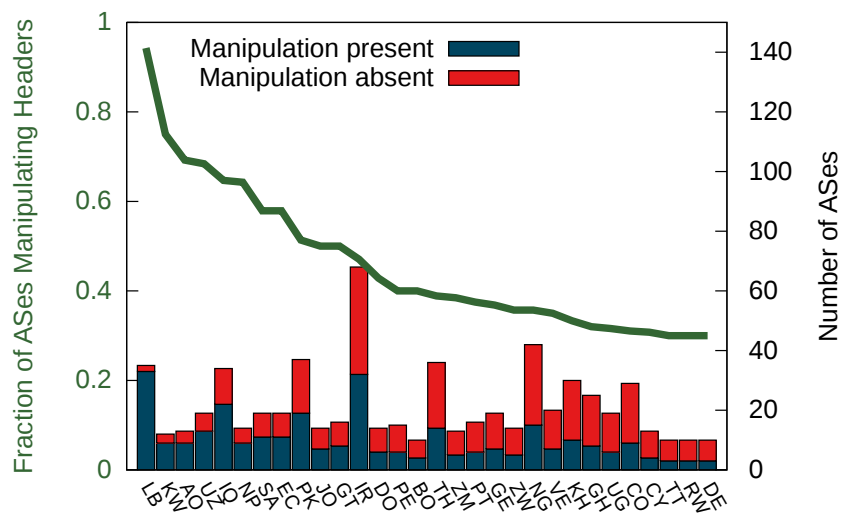

(a) Request headers

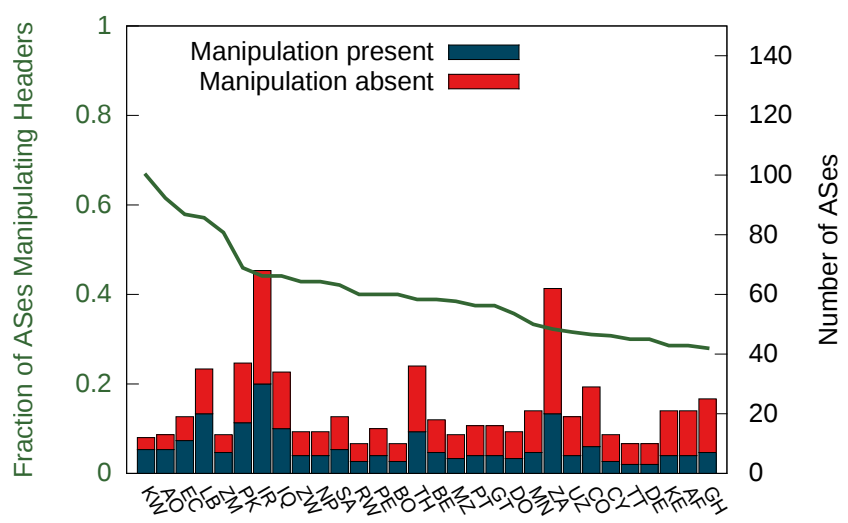

(b) Response headers

Figure 6: The bars show the number of ASes that modify $(a)$ request, and $(b)$ response headers per country. The green line shows the fraction of ASes that modify per country.

the veracity of our findings. The main reason listed by European operators was the progressive reductions in network transit prices, alongside greater peering via Internet eXchange Points [6]. In conjunction with higher line rates, this meant that such operators may actually have to pay more for running multi-Gbps web caches than simply contacting the origin via peering or transit. This, however, was not the case for African operators, who still complained of exorbitant transit costs and a distinct lack of peering [14]. Another frequently cited reason by European operators was the deployment of dedicated provider-specific caches in their networks (e.g., Google Caches, Netflix Appliances). Considering the bulk of traffic handled by these websites, the need for augmentary transparent caches was radically reduced. But, again, the presence of these in Africa is still limited [15]. These reasons have meant that the business case for transparent caching in developed regions has reduced, whereas it is still strong in developing countries.

\section{WHAT MODIFICATIONS ARE MADE?}

The previous section has shown how regions and networks differ in their frequency of header manipulation. Next, we inspect the content of the changes made. To explore this, we manually classify all manipulated headers into functional categories using various resources (e.g., RFCs, W3C, IANA, blogs). Table 2 presents the 5 categories, inclulding the number of injections/modifications we 


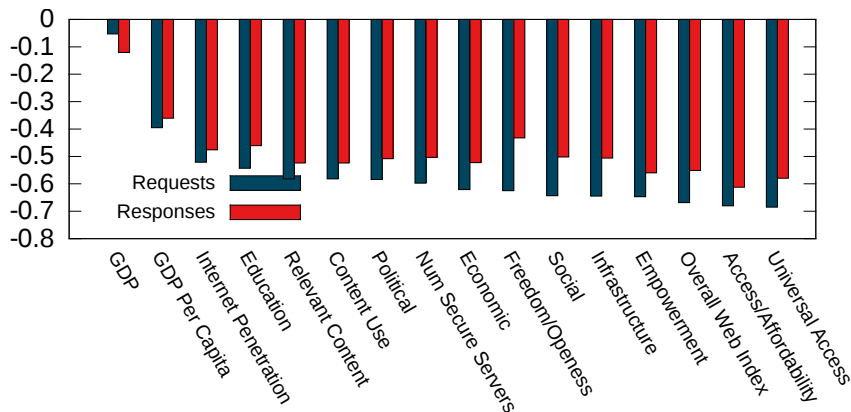

Figure 7: Correlation coefficient for fraction of ASes that manipulate in a country $v s$. metrics taken from the Web Index and World Bank (only countries with 10+ AS samples).

\begin{tabular}{|l|c|c|c|c|}
\hline \multirow{2}{*}{ Header Type } & \multicolumn{2}{|c|}{$\begin{array}{c}\text { \#Headers in } \\
\text { Category }\end{array}$} & \multicolumn{2}{c|}{$\begin{array}{c}\text { Total \#Headers } \\
\text { injected/modified }\end{array}$} \\
\hline & Request & Response & Request & Response \\
\hline \hline Cache & 4 & 9 & 8419 & 3799 \\
Operational & 12 & 9 & 5090 & 63 \\
Feature & 8 & 3 & 639 & 1884 \\
Information & 1 & 5 & 20 & 20 \\
Unknown & 4 & 3 & 10 & 41 \\
\hline
\end{tabular}

Table 2: Number of headers in each category, and number of instances of headers being injected/modified (count based on unique IP addresses).

see globally; a full list of the classifications can be found in [26]. Figure 8 separates all IP samples into their continents, and presents the fraction of manipulations that fall into each category. We emphasise that we are not trying to classify middleboxes themselves; instead, our focus is on the individual headers they manipulate. We now describe each category, and their regional presence.

\subsection{Caching Headers}

Caching headers are those that add information relating to cache operations (e.g., cache hits); these account for a significant fraction of manipulations. In-line with earlier discussion (\$5.2), we find that regions with higher transit prices tend to have a higher proportion of caching headers [6]. For instance, 93\% of IPs (79\% of ASes) sampled in Oceania (OC) witness cache-related response manipulations ( $\mathrm{OC}$ has amongst the most expensive transit in the world [6]); it is also common in other regions with high transit prices, i.e., Africa (AF), South/Central America (SA) and the Middle East (ME). In contrast, well-networked regions with reduced transit prices such as Europe (EU) and North America (NA), show the fewest cache-related manipulations. Figure 8 also shows that continents that see high proportions of cache-related response manipulations, also see similar trends for their requests. The only notable exception is SA, which sees a high proportion of cache headers injected into responses (79\% of IPs, $77 \%$ of ASes), but few in requests (17\% IPs, $18 \%$ Ases); the exact reasons for this trend are unclear. $^{4}$

Next, to inspect the specific header attributes, Figure 9 presents the global most frequently modified headers. Many at the top are cache related. This is largely because of a few specific headers that are regularly manipulated. $5 \%$ of measured ASes edit our preset

\footnotetext{
${ }^{4}$ We are not stating that SA does not have caches - only that it is not injecting a high proportion of cache request headers.
}

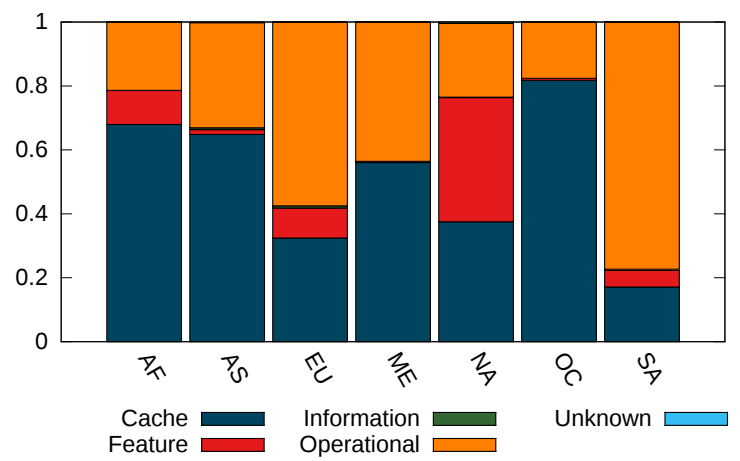

(a) Breakdown of request header types

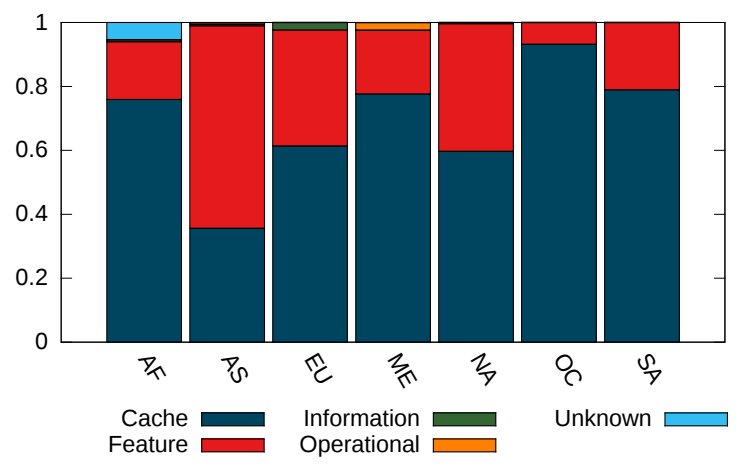

(b) Breakdown of response header types

Figure 8: Fraction of header manipulations in each category. Counts based on unique IP address samples.

Cache-Control response header. The majority of these changes (99\%) add extra settings (must-revalidate and no-store), whereas others change the existing ones, e.g., increasing the max-age setting from 0 to 60 . We also note that 3 ASes completely remove the Cache-Control header set by the server. This enables both browser and in-network caching against our will. Other prominently injected headers include X-Cache (9\% of ASes), and XCache-Lookup (6.9\% of ASes). These non-standard headers are used by a variety of cache implementations to report cache hits (making it trivial to detect caches). We note they may be used for attacks [16]. Their presence also allows us to check for middleboxes that might be caching uncacheable content against the instructions of our server. We find one South American AS doing this. Another cache-related header (injected in 18 ASes) is Age, which states the age of the object in the cache. 110 samples have this added, with 96 setting values greater than 0 , further revealing caches that store uncacheable content.

\subsection{Operational Headers}

Operational headers are those that pertain to infrastructural operations other than caching, e.g., tagging middleboxes, firewalls, recording IPs. Globally, we only observe 18 Middle Eastern, 6 Asian and 3 African ASes injecting operational response headers, all of which are non-standard (various bespoke functions, $e . g$., vendor specific firewalls).

Far more networks inject operational request headers though. In fact, in SA, the vast majority of request manipulations are operational. The high frequency is due to two key headers. The most frequently injected one is Via, as dictated by RFC 2616. This header 


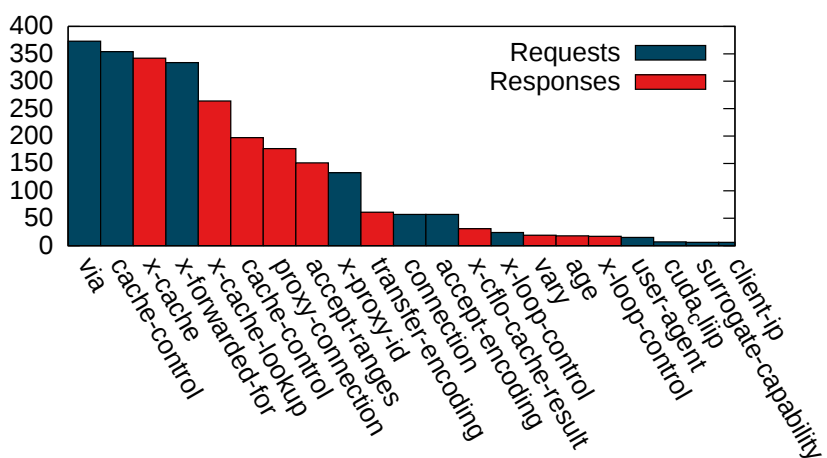

Figure 9: Number of ASes that manipulate each header.

is injected by a middlebox to inform the server of its presence. We observe this within $9.8 \%$ of ASes; perhaps more noteworthy is the remainder that do not implement this standard. This again confirms the poor adherence to standards exhibited by in-the-wild middleboxes.

The second most frequently injected operational header is $\mathrm{X}$ Forwarded-For, present in samples from $8.4 \%$ of ASes (not including vendor-specific equivalents like Client-IP and X-Real-IP). This header identifies the client IP address when being forwarded through a proxy. That said, in $64.7 \%$ of cases, this header stipulates a private address (e.g., 10.0.0.0/8), with a further $11.2 \%$ listing "Unknown", making it redundant for its purpose. We note that X-Forwarded-For has gained widespread usage, whereas the standardised version, Forwarded (RFC 7239), was not encountered a single time. This suggests that converting $X$ headers into standards is a non-trivial process. We also see a cellular-specific header worth mentioning: 18 IP addresses (within an AS in Jordan) inject the phone number of the mobile device using a header called MSISDN. ${ }^{5}$ This leak was also observed in [29], and certainly underlines the capacity for privacy undermining activity within these injections. Finally, we also see various bespoke operational headers from web filters/firewalls; e.g., Barracuda's CUDA_CLIP (7 ASes). We see no particular geographical pattern regarding these vendors.

\subsection{Feature Request/Advert Headers}

Feature headers are those that request or advertise a certain behaviour from the opposite endpoint. These mostly occur in responses, with Asia and NA injecting the greatest proportion overall. For example, Accept-Encoding and Connection are two preset feature headers that are manipulated (both by $1.5 \%$ of ASes). That said, it is actually more common to remove them: $12 \%$ of ASes completely remove the Connection header, disabling keepalive settings. Other middleboxes inject their own feature adverts to make it seem like the server has advanced functionality. For example, we find 6 ASes injecting the Surrogate-Capability header (this offers support for the Edge Side Language, which allows remote composition of websites on the client). There is one further feature header worth noting: 718 ASes remove the Keep-Alive header. RFC 2616 formalised the use of Keep-Alive, classifying it as a hop-by-hop header that should be removed by proxies. Clearly, many middleboxes adhere to this standard and, thus, it is an effective means to detect middleboxes.

\footnotetext{
${ }^{5}$ We do not see many cellular headers because Hola is aimed at desktop devices.
}

\subsection{Information Headers}

Information headers contain metadata that describes the client or server. Information headers are rarely seen in the data, with a slightly higher propensity to see them in developed regions: NA and EU. An interesting example is the User-Agent header, which informs the server of the type of browser requesting the page. We find 15 ASes manipulating this, and downgrading the browser version, e.g., from Firefox 5.0 to 4.0. We even see 378 IP addresses where the HTTP version is downgraded to 1.0 (from 1.1). In $82 \%$ of the samples, these requests had passed through a Squid proxy. Worryingly, we often see old middlebox software: $34 \%$ of Squid samples are running version 2.7 or older (last updated 2010). We even find 22 ASes using Squid software that has not been updated for at least a decade (v2.5). These are overwhelmingly in countries that rank lowly in the Web Index; apart from two ASes in Australia and Belgium, the highest ranked country is 32nd (Czech Republic).

Finally, we observed 28 responses in which a Set-Cookie header was injected. A Croatian AS was responsible for 8 of these, likely part of monitoring or customer tracking $[5,4]$. There were a further 20 samples that had cookies returned due to interceptions by various other types of middleboxes (e.g., Netscalar, Cisco Access Control). This actually highlights a particularly worrying feature of Hola, as it allows users to obtain the cookie identifiers of others.

\subsection{Unknown Headers}

It is worth briefly noting that we could not conclusively classify a number of headers: X-Client-TOS (4 ASes), SFID, X-TMV-Type (2 ASes), X-DG-TaggedAs, X-IMForwards (1 AS) and the enigmatic - - - - - (1 AS). The fact that no public documentation exists perhaps indicates that notable subsets of HTTP can no longer be considered "standard". The region with the greatest proportion of these is AF, although they also occur in NA and AS.

\section{RELATED WORK}

Middleboxes have recently become a hot topic. Early work highlighted their expanding and diverse roles [25], whilst recent work has been developing ways to more openly interact with them [20, 33]. Most related to our work is the small set of studies specifically targeting HTTP middleboxes. We have taken inspiration from the pioneering work performed by the Netalyzr service $[18,30]$, which crowd sourced measurements from volunteers. In 2010, they found that $8.4 \%$ of Netalyzr sessions passed via an HTTP proxy [18], whilst that increased to $14 \%$ in 2014 [30]. Another recent study quantified HTTP middleboxes in cellular networks $[29,28]$ showing that $13 \%$ (of 299 mobile operators) were manipulating headers [28]. Our work has confirmed a wide presence of middleboxes. Furthermore, unlike past work, our contributions have gone beyond detecting middleboxes: we have shed light on the different usages of middleboxes across types of networks and regions. Through this, we have explored the specific types of manipulations performed by these diverse regions, highlighting their relevance. It is also worth noting that another interesting study recently utilised the Hola infrastructure, although the focus was not on header manipulation [9].

\section{CONCLUSION}

This paper has explored the diverse HTTP header manipulations performed across regions and networks. Whereas past work has explored how these header manipulations reveal data about users, we shed insight on how they can expose network and regional trends. We find that header manipulation is commonplace: hosts in $25 \%$ of measured ASes witness headers modifications at least once. We also observe that middlebox injections are substantially different 
across regions. For instance, our results show that well connected regions such as Europe expose fewer caching headers than regions such as Africa, where transit is costly. While revealing, it should also be noted that exposing this information is a potential security threat, due to the frequent use of old and vulnerable middlebox software [7].

Our future work will focus on exploring how these trends evolve. Interestingly, many HTTP/2.0 browser implementations are following an encrypt everything model, which will undermine some middlebox functions. This is perhaps concerning as our work indicates a widespread dependence on their functionality. Hence, ISPs may endeavour to find ways around this [27]. This is particularly the case for security and performance oriented middleboxes, which may be considered critical to business operations. Hence, we believe that the continued monitoring of this process could offer fascinating insight into how network operators react and optimise to changes in Web protocols.

\section{REFERENCES}

[1] The CAIDA UCSD AS classification dataset. http: //www.caida.org/data/as_classification.

[2] Hola. https://hola.org/.

[3] Luminati FAQ. https: / / luminati.io/faq.

[4] Verizon injecting perma-cookies to track mobile customers, bypassing privacy controls. https: / /www.eff.org/ deeplinks/2014/11/verizon-x-uidh.

[5] AT\&T stops using invasive perma-cookies, but it may turn them back on.

http://www.wired.com/2014/11/att-hitspause-privacy-busting-perma-cookie-test, 2014.

[6] The relative cost of bandwidth around the world. https: / / blog.cloudflare.com/the-relative-costof-bandwidth-around-the-world, 2014.

[7] Squid: Security vulnerabilities. https: //www. cvedetails.com/vulnerabilitylist/vendor_id-823/Squid.html, 2016.

[8] Carlucci, G., De Cicco, L., And Mascolo, S. Http over udp: an experimental investigation of quic. In Proc. ACM SAC (2015).

[9] Chung, T., Choffnes, D., And Mislove, A. Tunneling for transparency: A large-scale analysis of end-to-end violations in the internet. In Proc. ACM IMC (2016).

[10] Detal, G., Hesmans, B., Bonaventure, O., VANAUBEL, Y., AND Donnet, B. Revealing middlebox interference with tracebox. In Proc. ACM IMC (2013).

[11] Dhamdhere, A., AND Dovrolis, C. Twelve years in the evolution of the internet ecosystem. IEEE/ACM Transactions on Networking (ToN) (2011).

[12] Dimitropoulos, X., Krioukov, D., Riley, G., Et Al. Revealing the autonomous system taxonomy: The machine learning approach. In Proc. PAM (2006).

[13] Elkhatib, Y., Tyson, G., AND Welzl, M. Can spdy really make the web faster? In IFIP Networking (2014).

[14] Fanou, R., Francois, P., And Aben, E. On the diversity of interdomain routing in africa. In Proc. PAM (2015).

[15] FAnou, R., Tyson, G., Francois, P., AND SATHIASEELAN, A. Pushing the frontier: Exploring the african web ecosystem. In Proc. WWW (2016).
[16] Huang, L.-S., Chen, E. Y., Barth, A., Rescorla, E., AND JACKSON, C. Talking to yourself for fun and profit. In Proc. Workshop on Web Security and Privacy (2011).

[17] KLYNe, G. Message headers.

http://www.iana.org/assignments/messageheaders/message-headers.xhtml, 2015.

[18] Kreibich, C., Weaver, N., Nechaev, B., And PAXSON, V. Netalyzr: Illuminating the edge network. In Proc. ACM IMC (2010).

[19] Naylor, D., Finamore, A., Leontiadis, I., Grunenberger, Y., Mellia, M., Munafò, M., PAPAgIANNAKI, K., AND STEEnKISTE, P. The cost of the $\mathrm{S}$ in HTTPS. In Proc. ACM CoNEXT (2014).

[20] Naylor, D., SChOMP, K., VARVEllo, M., LeOntiadis, I., BlackBurn, J., LÓPEz, D. R., PAPAgIANNAKi, K., Rodriguez Rodriguez, P., AND STEEnkiste, P. multi-context TLS (mctls): Enabling secure in-network functionality in TLS. In Proc. ACM SIGCOMM (2015).

[21] Poese, I., Uhlig, S., KaAfar, M. A., Donnet, B., And GUEYE, B. Ip geolocation databases: Unreliable? SIGCOMM CCR (2011).

[22] Sanchez, M. A., Otto, J. S., Bischof, Z. S., Choffnes, D. R., Bustamante, F. E., KRISHNAMURTHY, B., AND WILLINGER, W. A measurement experimentation platform at the internet's edge. IEEE Transactions on Networking (ToN) (2014).

[23] Scott, W., Bhoraskar, R., AND Krishnamurthy, A. Understanding open proxies in the wild. Technical Report. http://netlab.cs.washington.edu/squid/ paper.pdf, 2015.

[24] ShavitT, Y., AND Zilberman, N. A geolocation databases study. IEEE Journal on Selected Areas in Communications (2011).

[25] Sherry, J., Hasan, S., Scott, C., Krishnamurthy, A., RATnASAmy, S., AND SEKAR, V. Making middleboxes someone else's problem: network processing as a cloud service. SIGCOMM CCR (2012).

[26] Tyson, G. Dataset. http://bit.1y/1qg7pt4.

[27] Vallina-Rodriguez, N., Amann, J., Kreibich, C., WeAver, N., AND PAXson, V. A tangled mass: The android root certificate stores. In Proc. ACM CoNEXT (2014).

[28] Vallina-Rodriguez, N., Sundaresan, S., Kreibich, C., AND PAXSON, V. Header enrichment or ISP enrichment? Emerging privacy threats in mobile networks. In Proc. HotMiddlebox (2015).

[29] Vallina-Rodriguez, N., Sundaresan, S., Kreibich, C., WeAVER, N., AND PAXSON, V. Beyond the radio: Illuminating the higher layers of mobile networks. In Proc. MobiSys (2015).

[30] Weaver, N., Kreibich, C., Dam, M., and PaXson, V. Here be web proxies. In Proc. PAM (2014).

[31] Web index. http://thewebindex.org, 2016.

[32] World bank data repository. http: //data.worldbank.org, 2016.

[33] ZHOU, Z., AND BENSON, T. Towards a safe playground for HTTPS and middle boxes with QoS2. In Proc. HotMiddlebox (2015). 